\title{
De l'interaction en classe à l'action revécue : le clair-obscur de l'action enseignante
}

\author{
Francine Cicurel \\ Université Paris III - Sorbonne Nouvelle, DILTEC \\ Véronique Rivière \\ Université Lumière Lyon 2, ICAR UMR CNRS 5191 et associée DILTEC
}

Comment connaître une action d'enseignement? Est-ce par le biais de la description linguistico-discursive - minutieuse, à partir de transcriptions de séquences de classe ou en tentant de dégager progressivement ce qui apparaît comme récurrences, rituels, routines ? Ou peut-on faire le projet d'aller à la rencontre de ce qui est davantage enfoui, en essayant de faire émerger ce qui sous-tend l'action, la motive et la modifie? La description du discours et des interactions en classe, depuis près de trois décennies, a produit une riche série d'études prenant le discours de la classe comme objet de recherche. Les analyses de l'interaction in situ ont inauguré la possibilité d'une connaissance de la classe de langue à travers des descriptions fines de l'interaction en situation éducative (format des échanges, alternance des tours de parole, traces de l'objet à transmettre, actes de sollicitation, d'étayage, dimension métalinguistique, etc). Mais elles permettent peu d'avoir accès aux représentations des enseignants - la perception qu'ils ont de leur public, les idées et les conceptions qu'ils forment eux-mêmes à propos de l'action d'enseignement qu'ils ont à mener. Cet article se propose de se pencher sur l'apport que pourraient constituer l'adjonction et l'analyse d'autres sources pour comprendre l'interaction et, de ce fait, de réfléchir au statut de l'analyste chercheur et à celui du sujet observé. Il débouche sur une réflexion sur la complémentarité qu'il pourrait y avoir entre l'approche interactionnelle prenant comme objet central l'analyse des discours de la classe et les discours d'autocommentaires que peuvent tenir des professeurs de langue étrangère confrontés à leur propre pratique - quelle en est la nature, comment peuton les analyser? Sont-ils susceptibles d'apporter quelque chose de neuf, en particulier sur les processus interactionnels à l'œuvre dans une situation d'enseignement? Une analyse rétrospective faite par l'enseignant à propos de cours qu'il a donnés est-elle susceptible de donner un éclairage nouveau à la connaissance que nous avons de l'action didactique et de l'interaction?

\section{Propriétés d'une interaction didactique}

\subsection{L'analyse des interactions en classe de langue, hier et aujourd'hui}

Depuis le début des années 1980, que ce soit en contexte anglo-saxon ou francophone, un certain nombre de courants s'intéresse à la dimension interactionnelle des discours de classe de langue étrangère. Ces différents courants, par le biais de l'observation participante d'interactions "réelles", avaient d'abord une visée évaluatrice et transformatrice des situations éducatives et de leur efficacité (Flanders, 1960 ; Postic, 1977). Dans la tradition francophone, la priorité a d'abord été donnée à l'analyse des discours d'enseignement des langues (Coste, 1984 ; Ali Bouacha, 1986 ; Cicurel \& Moirand 1986 ) sur un plan énonciatif (traces de la position de l'autre dans le discours enseignant, polyphonie, double énonciation) et discursif. Par exemple, outre son caractère métalinguistique et autonymique, le discours de la classe de langue est parfois fictionnel lorsque l'enseignant éclaire le sens inconnu dans la 
langue étrangère (Cicurel, 1988). Ce sont par conséquent des traits définitoires relativement satisfaisants que l'on est parvenu à donner de la «didacticité » d'une interaction.

Puis, une dimension compréhensive et processuelle des faits d'interaction ${ }^{1}$ a été privilégiée (et continue de l'être), sous l'influence grandissante de l'interactionnisme social (ethnographie de la communication, sociologie goffmanienne, ethnométhodologie, analyse conversationnelle) importé en France par Kerbrat-Orecchioni $(1990,1992)$ en particulier. Elle consiste à observer et déterminer les mécanismes à partir desquels les participants à un événement communicationnel verbal parviennent, grâce à la mobilisation de ressources linguistiques et discursives, à coordonner leurs actions. L'interaction est décrite comme un phénomène d'ajustement réciproque dans une situation particulière, comme la classe de langue par exemple. De nombreux travaux, notamment en didactique des langues, s'attachent à mettre en lumière des phénomènes interactionnels très divers comme la structuration des échanges didactiques (Bouchard, 1984, 2005), l'établissement de la relation au cours des premières séquences de cours (Bigot, 2002), les modalités du dire de faire (Rivière, 2006), ou l'appropriation d'une compétence orale, écrite, lexicale.

De même, l'étude de la communication exolingue en lien avec la linguistique de l'acquisition des langues secondes a beaucoup appris, pour les situations formelles d'enseignement, sur la contractualité didactique et implicite des échanges exolingues (Matthey, De Pietro \& Py, 1989), sur les formes interactionnelles de l'interaction tutélaire comme la bifocalisation (Bange, 1992), sur l'activité métalinguistique (Ishikawa, 2002), ou encore sur les lignes de partage de l'asymétrie langagière et interpersonnelle (Vasseur, 2005). L'interaction est alors considérée comme constitutive des processus d'acquisition (Gajo \& Mondada, 2000).

\subsection{Un type interactionnel}

Inscrite dans ce champ des sciences du langage et de la recherche en acquisition des langues secondes, 1' 'analyse fine de l'interaction in situ a permis de dégager certains paramètres récurrents et typiques de l'interaction d'enseignement. Cela a conduit à considérer l'interaction didactique comme un type interactionnel ayant ses règles propres de fonctionnement, différentes de celles qui ont cours dans une conversation familière ou amicale, ou une négociation commerciale. Essayons d'en repérer quelques-unes à la lumière d'une analyse empirique.

Une enseignante expérimentée de français langue étrangère, surnommée Catherine, s'adresse à un public chinois dans une université française ${ }^{2}$. Elle est en train de mener dans cette séquence de classe une activité grammaticale sur le subjonctif dans un cadre communicatif. Chacun doit produire à tour de rôle un énoncé sur le modèle «j'aime/je n'"aime pas » en utilisant une proposition complétive au subjonctif ou à l'infinitif, ceci dans le but de pouvoir formuler une règle d'utilisation.

\footnotetext{
${ }^{1}$ Voir l'important courant de la classroom discourse analysis avec Allwright (1988) et Van Lier (1988) par exemple.

${ }^{2}$ Le dispositif de recherche a été mis en place par Hélène Ginabat (que nous tenons à remercier pour la constitution du corpus sur lequel travaille actuellement le groupe «agir professoral» du DILTEC) et exposé dans son mémoire de Master 2 Recherche. Le corpus est constitué de cours filmés et transcrits et de commentaires de l'enseignante sur le filmage de sa classe, eux aussi transcrits. L'entretien, en situation d'autoconfrontation simple, a été réalisé et enregistré un mois après le filmage des cours. Le début de l'entretien est guidé par le chercheur qui a présélectionné les moments d'arrêt de l'image et de commentaire. Puis, rapidement, l'enseignante prend le relais et interrompt la lecture vidéo quand elle le juge bon. Au moment de l'interruption du film, les commentaires de l'enseignante peuvent porter sur ce qui vient juste de se passer, ou sur un événement un peu plus lointain.

Conventions de transcription $:=$ apprenant. + et $++=$ pause. MAJUSCULE $=$ mot ou syllabe accentué. $::=$ allongement syllabique. $($ rire $)=$ commentaire du transcripteur sur l'activité non verbale. $\uparrow=$ Intonation montante. Les éléments que l'on veut mettre en valeur sont en caractère italiques. $(E)=$ moment où l'enseignante stoppe la bande filmée pour commenter son action.
} 


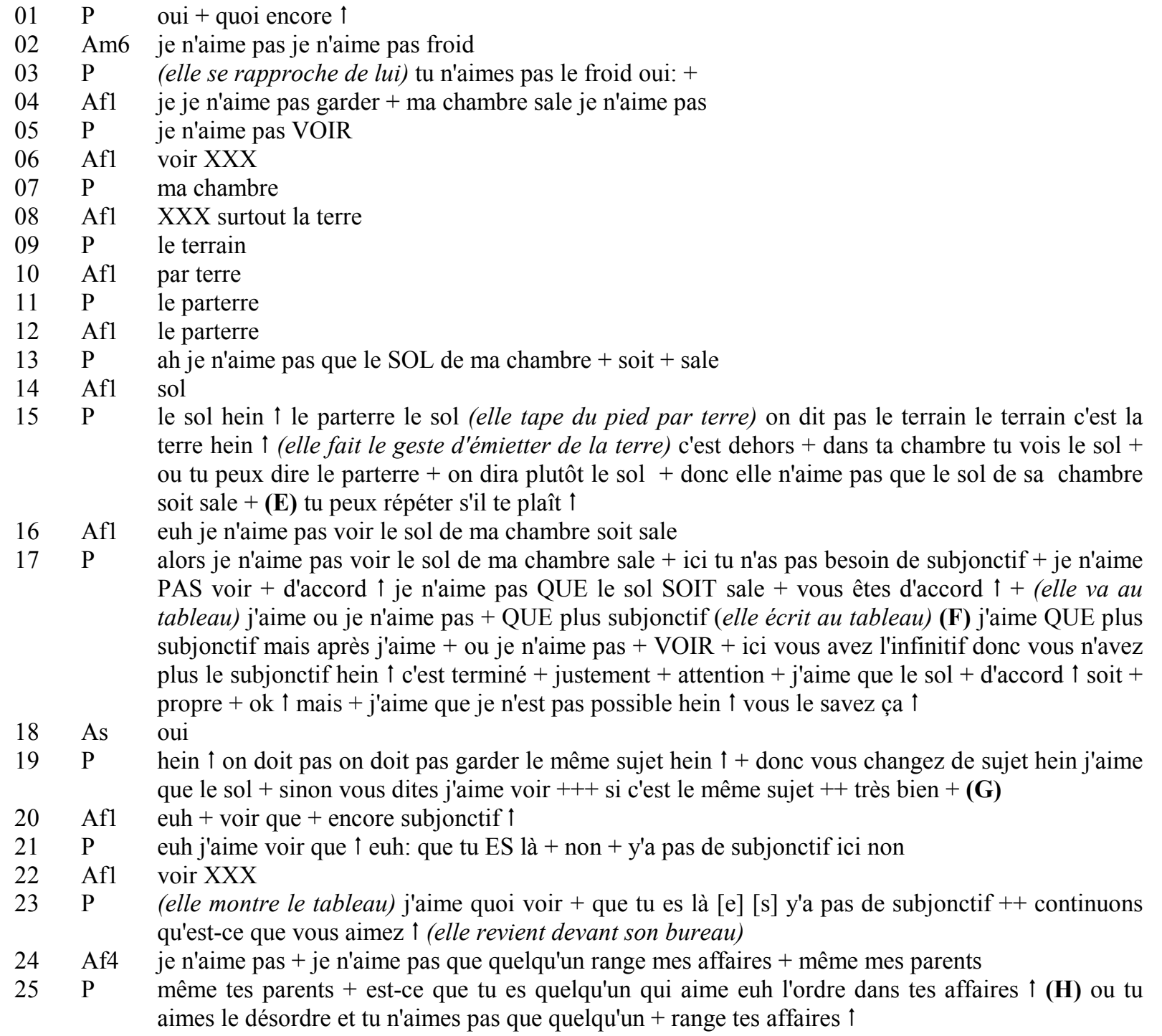

Cet échange, de nature polylogale (tout le monde peut participer), mais mené sur le mode dilogal (P-A), permet d'entrevoir la manière ou la «méthode» par laquelle l'enseignante organise et gère le contexte cognitif de l'activité. En effet, l'activité, déjà sur les rails, consiste à ce que chaque apprenant produise un énoncé selon un certain rythme. Lorsque celui-ci est ralenti, l'enseignante relance et cède l'initiative à un apprenant (01 et 02$)$. Mais cette activité provoque aussi une intense activité de reprise et de reformulation $(05,13,17)$, d'achèvement interactif $(07)$, de réparation $(09,11)$, en même temps que l'enseignante fournit les ressources linguistiques pour la production et l'énoncé. C'est à deux qu'un énoncé se construit petit à petit.

On est ainsi dans l'ordre tranquille et ritualisé de l'interaction didactique en L2 où l'enseignante a un projet précis (fournir une règle à partir des productions spontanées). Cela l'amène à laisser une certaine initiative dont elle a besoin pour parvenir à ses fins. Mais elle contrôle très étroitement les prises de parole des apprenants. Ceux-ci peuvent toutefois déroger à ce contrôle, comme dans le TP 20 où l'apprenante demande un complément d'information grammaticale : elle semble vouloir vérifier si «voir que » peut fonctionner aussi avec le subjonctif. L'enseignante offre ce complément qui ne semble pas satisfaire l'apprenante en 22, puisqu'elle renouvelle sa question. Mais en 23, l'enseignante redit à peu de chose près ce qu'elle a proposé en 21 et réoriente ensuite verbalement et par un 
déplacement physique le topic en poursuivant l'activité. On a donc affaire à ce que Py (1993) appelle une «apprenante curieuse» qui investit son «territoire d'apprenante ${ }^{3}$ » par «la manifestation d'un comportement plutôt exploratoire ou ludique face à la langue, le plaisir de manipuler des formes l'emporte sur le souci de la conformité sociale et de l'efficacité communicative » (p. 21). Elle donne à voir la manière dont elle règle et développe sa compétence dans la langue cible, le travail émergent d'appropriation de savoirs linguistiques et de savoir-faire métalinguistique. De son côté, l'enseignante reste focalisée sur la réalisation de la tâche. Si l'apprenante semble focalisée sur le système, l'enseignante ${ }^{4}$ semble l'être sur la tâche.

L'analyse nous montre ainsi les procédures conjointes mises en œuvre, la négociation et la ratification des savoirs linguistiques.

Cet extrait, replacé dans le corps constitué des études empiriques existantes, permettrait d'esquisser certaines spécificités de l'interaction didactique (Cicurel \& Doury (Ed.), 2001) comme :

- le maintien de rôles et places d'expertise/non expertise (le rapport asymétrique au savoir est une condition de l'interaction didactique); on observe couramment les efforts faits par les interactants pour défendre leur place respective ;

- un cadre spatio-temporel prédéfini (un lieu fixe, des contraintes horaires que connaissent les participants) qui est généralement une institution à but éducatif ;

- une forte inscription de l'interaction dans un en-dehors constitué par un ensemble de textes écrits (programmes, manuels, notes de préparation de cours) qui précèdent presque toujours l'interaction et qui lui succèdent (écrits liés à l'évaluation) ;

- une visée cognitive, se manifestant notamment dans le cas d'une classe de langue par la présence d'activités pédagogiques constituées dont le but est d'encadrer l'apprentissage ;

- une constante dimension évaluative de la part des enseignants destinée à vérifier l'efficacité de la transmission, il faut qu'il y ait progrès dans le savoir des participants.

Si les situations éducatives dans leur diversité ne se ressemblent pas sur tous les points, elles mettent toutefois en œuvre les éléments précités.

\section{Qui observe qui et quoi?}

L'analyse interactionnelle d'un événement didactique s'inscrit dans une linguistique interprétative, et les différents acquis énumérés (1.1) et l'analyse empirique esquissée (1.2) partagent, sur le plan méthodologique, au moins deux principes.

\subsection{Statut de l'observation}

Le premier principe concerne la prise en compte du contexte et des acteurs d'une interaction. Il s'agit de comprendre et de donner à voir leurs "méthodes », leurs modes d'interaction au sein du contexte et la manière dont ils les orchestrent. Pour cela, le principe méthodologique d'indexicalité consistant à " reconnaître que l'observateur s'intègre dans le contexte observé » (Mondada, 2001, 3) prévaut. Pour autant, l'acteur de la situation n'est pas sur le même plan que l'observateur-chercheur. C'est ce que nous rappelle Schütz (1987), dans une perspective phénoménologique et sociologique de l'interprétation de la réalité, lorsqu'il réfléchit au statut de l'observation et aux compétences de l'observateur en s'appuyant sur la métaphore de l'individu dans la ville. Un citadin s'oriente naturellement dans un espace urbain familier, il a

\footnotetext{
${ }^{3}$ Le territoire de l'apprenant correspond au «contexte linguistique dans lequel se développe les processus d'acquisition d'une langue étrangère. »(p. 9). Ce territoire est défini par 3 pôles par rapport auxquels les apprenants ont à se situer et qui contribuent en quelque sorte à délimiter leur profil d'apprentissage : «la construction d'un système de connaissances linguistiques; l'ajustement aux normes de la langue cible et l'accomplissement de tâches spécifiques $\gg$.

${ }^{4} \mathrm{Si}$ on peut postuler à cet égard que l'enseignant a lui aussi un territoire d'enseignement.
} 
le sens de la distance entre les lieux, il possède une représentation du temps que prendra tel ou tel trajet. Sa connaissance est non systématique, mais ce savoir pratique lui suffit. A cet effet, le professeur qui possède une certaine expérience, qui fait son cours depuis un certain temps, est à comparer à ce citadin, habitant à l'aise dans sa ville. Il connaît ses élèves, il sait ce qu'il a à faire et met en place de moyens d'enseignement qu'il maitrise. A travers des transcriptions provenant d'observations de ce professeur dans sa classe, nous pouvons le voir à l'œuvre « dans sa ville » et identifier quelques-unes de ses pratiques didactiques.

Toute autre est la stratégie d'orientation que doit mettre en place un étranger arrivant dans une ville pour la première fois. Rien ne va de soi, il est tenu de mettre en place des stratégies " conscientes " pour s'orienter, se servir d'une carte, demander son chemin, etc. Enfin le rôle du scientifique, le cartographe, intégré lui aussi à la ville, qui, en l'occurrence, ne va pas se limiter à la collecte d'informations auprès des habitants, même dotés d'un solide sens pratique, est de développer des méthodes propres à la science cartographique. C'est le rapport entre l'habitant compétent et le scientifique qui est ici intéressant: les connaissances du chercheur et de l'acteur ne sont pas élaborées et restituées de la même façon, n'ont pas les mêmes finalités ${ }^{5}$.

\subsection{Une perspective située de l'interaction?}

Le second principe est la primauté accordée aux données authentiques et contextualisées, qu'elles soient orales ou non. Cela implique qu'elles soient «recueillies » dans le milieu où elles ont été produites, sans qu'elles aient été provoquées par telle ou telle procédure de contrôle ou de sélection. Le travail à partir de ces données peut donner l'illusion de « capturer le réel » : puisqu'on a filmé, enregistré, transcrit et que l'on est devant des dires effectivement prononcés, c'est de la «réalité » qu'il s'agit. Toutefois, rappelons-le, la classe n'est pas $a$ priori un objet de recherche, c'est d'abord un lieu de travail où on apprend et où on enseigne. S'y entrecroisent des forces provenant de sources différentes : interactions verbales, programmes, résultats, méthodologies, passé et buts des acteurs, lieux institutionnels, enjeux symboliques, système de places, tout y est. Dans ces conditions, à quels moyens faut-il faire appel pour parvenir à transformer le vivant en objet de recherche? La constitution de données pour la recherche ne donne pas à lire directement la réalité d'une interaction, mais s'appuie sur un processus de transformation et de médiation de ces données (Rivière, 2006), à travers le filtre du moyen technique adopté pour le recueil, puis à travers l'opération de transcription de données sonores et visuelles, à travers un certain nombre de choix que le chercheur est amené à faire. De même, le temps de l'analyse n'est pas le temps de l'action elle-même, les différents modes de contacts ne peuvent tous être pris en compte ; le canal visuel, les gestes, l'intonation, le rythme, le ton, etc. sont difficiles à noter ${ }^{6}$. Mais au moins, le but reste celui de décrire au mieux ce qui se passe dans une classe entre les participants en tenant compte du but qui les réunit.

\subsection{Lorsque l'acteur devient observateur de sa propre action}

Si le processus de construction de la recherche permet à un certain degré d'être "à l'aise " avec les pratiques didactiques observées, le chercheur reste tout de même aveugle sur certains aspects des processus interactionnels à l'œuvre. On le constate assez nettement lorsqu'on suscite, à travers un dispositif d'auto-confrontation simple (Amigues, Faïta, Saujat, 2004 ;

\footnotetext{
${ }^{5}$ Schütz $(1987,48)$ nous dit que l'observateur analyse son action à partir d'un « ici », à partir de « sa situation biographique propre », mais le scientifique n'a pas d'《ici ». Nous pourrions objecter cette dernière proposition. Le scientifique a bien un « ici », mais d'une autre sorte, son point de vue et son but n'étant pas le même que le praticien.

${ }^{6}$ On s'en aperçoit quand on lit une transcription et qu'on regarde ensuite la séquence filmée : quelque chose du réel ne peut pas être « imaginé » par le biais de la seule transcription.
} 
Clot \& al., 2001), des commentaires a posteriori ${ }^{7}$. Face au visionnement de la séquence de classe analysée en 1.2 ci-dessus, l'enseignante C. fait ce commentaire juste après le TP 15 de l'interaction de classe.

I qu'est-ce qui s'est passé là $\uparrow$

$\mathrm{P} \quad$ (E) ben là il s'est passé que elle avait du mal à formuler ce qu'elle voulait dire et que pas à pas j'ai devancé sa pensée et j'ai je l'ai aidée à formuler et peut-être qu'elle a eu l'impression qu'elle se débrouillait pas mal et tant mieux comme ça je l'ai accompagnée et ça lui donne confiance surtout cette élève-là qui est quelqu'un qui est très timide ça m'étonne qu'elle parle + c'est la 4ème qui parle ou quelque chose comme ça ça m'étonne qu'elle intervienne aussi vite parce que normalement c'est une très bonne étudiante très formelle mais qui à l'oral a une timidité euh et donc euh je l'ai vraiment accompagnée pour qu'elle puisse prendre confiance en elle là c'était typique hein du truc et j'ai aussi bon ben fait la différence entre le terrain et le sol le terrain à l'extérieur et le sol puisqu'elle avait + fait une confusion dans l'utilisation du vocabulaire + et ça ça vient du fait qu'ils utilisent le dictionnaire franco-chinois et en plus leur dictionnaire électronique là qui ne donne pas des définitions précises mais seulement des traductions

Elle semble insister sur l'organisation d'un contexte plus psychologique ou psychoaffectif que cognitif. Elle identifie une difficulté linguistique (« elle avait du mal à formuler »). Elle offre une aide cognitive et psychologique («pas à pas j'ai devancée sa pensée, je l'ai aidée à formuler, je l'ai accompagnée et ça lui donne confiance », «je l'ai vraiment accompagnée pour qu'elle puisse prendre confiance en elle »).

Mais il s'agit d'une organisation psychologique de l'interaction qui concerne cette apprenante-là, compte tenu de son profil psychologique identifié («très bonne étudiante très formelle, mais timide à l'oral »). La gestion de l'interaction de tutelle répond à une intention de mise en confiance pour une meilleure efficacité communicative et s'adapte au profil de l'apprenant.

Catherine fait ainsi part des préconstruits ou des «prémisses organisationnelles » (Goffman (1991) de l'interaction à partir desquels elle gère cette interaction et le savoir : l'apprenante, son profil et ses conduites qu'elle a identifié, catégorisé préalablement, dans l'expérience commune à ce groupe-classe.

De même, à propos du territoire de l'apprenante et de son initiative, voici ce que verbalise C. après le TP 25 :

P (H) en fait quand j'ai donné l'explication donc là ils m'ont poussée encore plus loin + avec cette classe c'est très spécial normalement on s'arrête là + ils m'ont poussée plus loin et là j'ai stoppé sinon ils me poussent les Chinois + ils me poussent encore à faire de la grammaire et si j'avais dit ah ben là on s'arrête et on va faire un exercice de grammaire ils auraient été aux anges + il faut faire attention à ça + parce que eux ils ont toujours ce réflexe + et puis aussi donc y'a un peu épater les copains etc on va toujours plus loin dans les questions alors là moi j'ai stoppé + parce que pour moi cette question-là elle était pas au programme ce jour-là et que j'allais pas commencer à leur donner des explications plus loin on n'était pas dans un exercice de grammaire + mais ils sont toujours en train de nous titiller dès qu'on fait un exercice oral il faut qu'il y ait une question de grammaire + bon c'est bien mais on va pas plus loin on répond tranquillement mais on ne va pas plus loin parce que là on n'est pas dans la grammaire + n'oubliez pas + enfin je l'ai pas dit mais ça c'était flagrant que on revient à notre activité orale + on ne va pas n'essayez pas de me faire diverger vers autre chose + et les Chinois c'est les spécialistes pour nous faire diverger vers la grammaire hein

Elle semble y voir une tentative de déplanification («normalement on s'arrête là, ils m'ont poussé, j'ai stoppé »), de détournement de la tâche de systématisation de l'emploi j'aime/je n'aime pas » pour une activité focalisée sur le système. Là encore, elle s'appuie sur une certaine connaissance des conduites des apprenants chinois (« « ils me poussent les Chinois»,

\footnotetext{
${ }^{7}$ Le procédé méthodologique de l'entretien en auto-confrontation consiste à confronter l'acteur à son activité par le visionnement filmé de cette activité et un entretien avec un chercheur. Il s'agit dès lors de données provoquées, dont le statut est encore différent des données in situ. Le statut de l'enseignante s'en trouve également modifié. Dans l'auto-confrontation simple, «le sujet devient lui-même un observateur extérieur de son activité en présence d'un tiers » (Clot, 2001).
} 
«ils ont toujours ce réflexe», "épater les copains », "ils sont toujours en train de nous titiller ») qu'elle a bâtie, au fil des histoires interactionnelles vécues avec ce type de public, et qu'elle généralise de ce fait. Elle a aussi construit un certain nombre de réponses à apporter à ces comportements qu'elles soient effectivement données dans l'interaction ( « on répond tranquillement »), qu'elles soient de l'ordre du possible («si j'avais dit on fait un exo de grammaire, ils auraient été aux anges ») ou de l'impossible («c'était pas au programme à ce moment-là »). Par ailleurs, on peut supposer que C. construit plus ou moins consciemment, une image positive d'elle-même et de son action, et correspondante à l'image qu'elle se fait des acteurs commanditaires de cette enquête (l'informateur et les universitaires-chercheurs en didactique du FLE). Schütz (1987) souligne à cet égard que les interprétations d'expériences vécues varient en fonction du moment de cette interprétation et des intérêts de ce moment. Sur le plan de l'analyse, une précaution s'impose : le dispositif réflexif pèse de tout son poids sur les interprétations de C.

C. donne à voir le cadre d'interprétation a priori qu'elle avait posé pour cette situation d'interaction : l'initiative est acceptée uniquement pour produire un énoncé-type. Toute autre tentative, toute "transformation du cadre » sera repoussée, compte tenu du profil des élèves, de son projet.

De cette façon, l'enseignante dans son auto-confrontation fait émerger plusieurs aspects, qui apparaissent peu à l'observateur au moment de l'interaction in situ, celle qui se déroule en classe. Elle s'arrête notamment sur ses tactiques ("je l'ai accompagnée »), sur ce qui la surprend («ça m'étonne ») dans l'interaction, montrant l'attention qu'elle porte au public ou plutôt à chacun des participants-apprenants, elle se réfère à son expérience passée et fait sentir qu'elle décide de l'orientation de l'action en fonction de ce qu'elle sait déjà, de son « répertoire didactique ${ }^{8}$ ». Enfin, elle manifeste une certaine impatience lorsque les étudiants tentent de la faire dévier de son projet initial, plus « communicatif que grammatical».

\section{4 Étendre le domaine de l'" interaction réalisée » au "réel de l'interaction »?}

Alors que le point de vue de l'acteur et du chercheur semblait se confondre dans l'analyse de l'interaction effective, l'introduction d'un discours sur cette interaction permet de les confronter, de les faire dialoguer. C'est que nous enseignent notamment les travaux en ergonomie (Guérin \& al. 1997) ou en analyse de l'activité (Clot, 2001). Puisque l'action (d'enseignement) n'est pas directement observable et que certains mécanismes psychologiques propres aux acteurs demeurent opaques à l'analyste, il est possible, sous certaines conditions, de faire appel aux verbalisations et à l'implication de l'acteur pour relater son agir propre. Il s'agit alors de considérer l'interaction de classe comme « réalisée » et d'étendre celle-ci à l'étude du «réel de l'interaction » (les motifs, les représentations, les difficultés ressenties, etc.) pour reprendre une formule de Clot (1999) à propos de l'activité. Mais quelle est la distance susceptible d'exister entre le dire au cours de l'interaction didactique elle-même et le dire sur le faire et qui intervient a posteriori devant ce qui devient presque une mise en spectacle ? Il importe de prendre en compte le fait que la collecte des données ne se fait pas de la même manière. Dans le premier cas, selon une méthodologie à rapprocher de celle qui est propre à l'ethnographie de la communication, le chercheur se rend sur le terrain et recueille des interactions dans un contexte social donné (qui est ici un site scolaire). Dans le second cas, nous sommes sur un autre lieu et dans un autre temps que celui

\footnotetext{
${ }^{8}$ La notion de répertoire didactique a été forgée au sein du groupe de recherches sur les interactions en classe du Centre de recherches DILTEC. Elle réfère à l'ensemble des ressources que possède un enseignant. Tout comme le sujet parlant dispose d'un répertoire verbal dans lequel il puise, le sujet enseignant dispose d'un ensemble de modèles d'actions, de sommes d'expériences, de convictions qu'il mobilise à l'occasion de sa pratique d'enseignement (Bigot \& Cicurel (Ed.), 2005). Pour une définition plus complète, voir Cadet (2004).
} 
de l'interaction en classe ${ }^{9}$. Il s'agit d'une situation revécue ${ }^{10}$, verbalisée par l'acteur au cours d'un entretien à deux interactants.

S'intéresser à l'action humaine et à ses motifs est le fait d'un certain nombre de courants en philosophie, en sciences de l'éducation (Habermas, Ricoeur, Friedrich, Bronckart entre autres). Dans la veine sociologique, Lahire (2001) distingue deux grandes tendances dans les théories de l'action et de l'acteur. Un premier groupe de chercheurs analyse des moments d'action ou d'interaction en privilégiant l'ordre de l'interaction, les phénomènes interactionnels par lesquels les locuteurs entrent en contact, co-traitent le thème, s'accommodent des rituels (cf. section 1.1 ci-dessus). Un second courant s'intéresse davantage au passé et à l'expérience de l'acteur (théories de l'habitus, théories psychologiques). Pour Lahire, dans la lignée des travaux de Halbwachs, il est important de donner aussi sa place à la mémoire, au "passé incorporé » pour comprendre quelque peu l'action humaine. Les acteurs agissent en fonction de schèmes intériorisés, qui constituent le répertoire actionnel, le stock dont ils disposent.

Enfin, en phénoménologie sociale, Schütz $(1987,1998)$ propose une théorie de la manière dont s'édifient, se stabilisent et aussi se transmettent les significations sociales, via des processus d'interaction. Il affirme l'illusion d'un accès direct au réel et l'importance des activités pré-attentionnelles et antéprédicatives dans la compréhension d'une action sociale. Il donne ainsi toute sa place à l'expérience vécue, et «toute interprétation est [alors] fondée sur une réserve d'expériences antérieures, qui sont nos propres expériences et celles transmises par nos parents et nos professeurs. Elles fonctionnent comme un cadre de référence sous la forme d'une 'connaissance disponible'. » (1987, p.105). C'est qu'il nomme les typifications. Mais Schütz met également l'accent sur la structure temporelle de l'action et sur la confusion résultant de l'utilisation indifférenciée de "parce que » comme moyen de référer à ce qui relève de la projection dans le futur de l'action par l'acteur, et ce qui est à rapporter au passé. La distinction qu'il fait entre ces deux strates de l'action se conçoit en termes de différenciation de motifs : motif-en-vue de (la fin pour laquelle l'action est entreprise) et motif-parce-que de l'action (renvoyant à des expériences passées qui, d'une certaine manière, détermine l'agir présent). La première catégorie se rapporte à la subjectivité du sujet et renvoie aux intentions d'une action. Le motif-en-vue-de s'inscrit dans une projection dans le futur et est donc tourné vers l'avenir. La seconde catégorie se situe plutôt du côté de l'objectivité et réfère à quelque chose qui a déjà eu lieu.

Ainsi nous aurions deux versants de l'approche de l'action, l'un plus interactionnel et qui s'attache aux pratiques effectives d'un sujet incarnant un rôle social, et l'autre, plus enfoui, mais qui apparait nettement lorsqu'on interroge l'acteur professeur et qu'il évoque les motifs sous-jacents au discours. C'est sur cette dualité que nous avons forgé la notion d'agir professoral. Une interaction située d'enseignement prend corps dans une expérience sociohistorique de l'action.

\section{Agir professoral et intérêt pour les motifs de l'action}

L'«agir professoral » correspond à l'ensemble des actions verbales et non verbales, préconçues ou non, que met en place un professeur pour transmettre et communiquer des savoirs ou un "pouvoir-savoir » à un public donné dans un contexte donné (Plazaola Giger, 2007). Le professeur exécute une suite d'actions en général coordonnées, et parfois

\footnotetext{
${ }^{9}$ Dans le cas présent, c'est un mois après, au domicile de l'enquêtrice, que se déroule l'auto-confrontation.

${ }^{10}$ En effet, comme le note Clot $(2001,255)$, citant Vygotski, «l'action passée au crible de la pensée se transforme en une autre action, qui est réfléchie ». Mais il met également en garde contre les «pièges du vécu » et l'impossibilité d'« être en contact immédiat avec l'expérience du sujet ».
} 
simultanées, subordonnées à un but global, avec une certaine intentionnalité. Cette mise en action rencontre les dires des apprenants et doit s'adapter à eux.

L'agir enseignant possède la particularité de provoquer d'autres actions de la part du groupe d'apprenants puisqu'il faut provoquer des transformations de savoirs et parfois de comportements. Pour connaître l'agir professoral, il semble qu'il faille s'appuyer aussi sur l'explicitation que donnent les enseignants à propos de leur propre action d'enseignement.

\subsection{Interaction entre l'action et le commentaire sur l'action}

Nous avons abordé rapidement dans la section 2.3 ci-dessus le rôle des pré-construits ${ }^{11}$ dans l'interaction revécue par l'enseignante. Nous souhaitons y revenir et montrer de quelle manière nous pouvons comprendre ces «principes d'organisation" et le système de représentations complexes construit par $\mathrm{C}$, le rôle de l'expérience et des motifs cachés de l'interaction, dont nous ne percevons que le résultat dans l'observation de la classe. C'est, selon nous, par la dynamique du «dire sur» et de la pensée de l'enseignante, quand elle interagit avec, réorganise et reconstruit l'action vécue et réalisée, en donne une logique composite. Par exemple, dans ce commentaire

(E) oui c'est embêtant ça je suis obligée absolument de me gendarmer bon ben parce que c'est facile pour beaucoup + comme Zhenyang est quelqu'un qui a tout pigé déjà euh depuis longtemps euh et ben elle prend sa chance à chaque fois un peu + et même si je lui dis essaye Zhenyang je sais que tu peux répondre mais essaye quand même de te taire ça c'est le gros problème de cette classe hein c'est pour ça que je donne le ton un peu euh: agacé parce que: ça + ils ils se laissent aller par rapport à ça hein + dans cette classe c'est le problème + j'ai du mal à gérer la prise de parole

C. fait part d'une tension qu'elle vit dans le quotidien de sa pratique, entre son attente d'une prise de parole collective partagée (action voulue) et la réalité d'une prise de parole individuelle dominante, et qu'elle catégorise comme un "gros problème ». Mais elle fait part également de la manière dont elle tente de réguler cette tension, soit en adoptant un ton directif pendant le déroulement de son action et en ayant conscience, au moment de l'entretien, qu'elle doit «se gendarmer» dans ce type de situation, soit virtuellement par un discours qu'elle pourrait adresser à un apprenant trop loquace (comme Zhenyang ici à qui C. ne s'adresse pourtant pas dans les interactions effectives) et qu'elle met en scène en s'adressant en «je » à un «tu » («je sais que tu peux le faire mais essaie de te taire »). Dans les deux cas, la stratégie adoptée s'appuie sur une connaissance et une anticipation de la « réactivité » des apprenants.

La dynamique discursive du «discours sur» engage tout autant l'interaction non réalisée, empêchée ou hypothéquée que l'action réalisée (Clot, 2001). C. ne relate pas seulement une action passée, mais en donne des traces encore vivantes (en se projetant dans un présent de l'interaction par ses répliques).

Le déroulement de l'interaction «réalisée » ( $\mathrm{P}$ fait un rappel des étapes nécessaires à la rédaction d'un écrit) correspondant au commentaire (E), présentée ci-dessous,

$\mathrm{P}$ quelles sont les étapes SYSTEMATIQUES c'est à dire OBLIGATOIRES (elle écrit) + on va pas commencer à sauter une étape + d'accord $\uparrow$ c'est une méthode de travail je vous l'ai déjà dit d'accord $\uparrow$ on fait SYSTEMATIQUEMENT cela pour ne pas oublier ok $\uparrow$ (C) alors étapes systématiques ok systématiques c'est à dire obligatoires hein $\uparrow+++$ pour arriver (D) à écrire notre sujet quelle est la première étape $\uparrow$

Af analyser le sujet

$\mathrm{P} \quad$ (elle écrit au tableau) analyser le sujet qu'est-ce que ça veut dire analyser le sujet $\uparrow+$ et et y'en a pas qu'une seule qui sait ça hein dans la classe + alors on se réveille + tout le monde participe + ok $\uparrow$ ne comptez pas toujours sur Damian pour vous aider + c'est trop facile + alors qu'est-ce que ça veut dire analyser le sujet $\uparrow$ (E)

\footnotetext{
${ }^{11}$ La définition de Goffman $(1991,19)$ des «prémisses organisationelles » de l'action sied tout à fait à celle que nous pourrions donner à la notion de pré-construit : « des principes d'organisation qui structurent les événements - du moins ceux qui ont un caractère social - et notre propre engagement subjectif. »
} 
ne peut permettre cette reconstruction. Nous accédons davantage au résultat du processus qui a conduit à prendre, dans le vif de l'interaction, telle ou telle décision. On comprend la nécessité de convoquer le « discours sur » l'interaction et d'en analyser les ressorts.

\subsection{Les motifs de l'action enseignante}

Dans la dynamique interprétative de l'action, l'un de ces ressorts tient dans le fait que C. motive son action. Nous pouvons à ce sujet convoquer la distinction entre motifs-en-vue-de et motifs-parce-que faite par Schütz (cf. 2.4).

Par exemple, dans les commentaires portés sur l'activité grammaticale liée à la construction du subjonctif, voici ce que C. dit à partir du tour de parole $19(\mathrm{G})$ :

(G) alors là en fait ce qui se passe c'est que là on part d'une application pratique pour expliciter un point qui est très particulier euh le fait que on mette pas le même sujet dans la principale et la subordonnée (motifs en-vue-de) normalement je n'utilise pas ça pour introduire ce point-là qui est important + euh mais là j'ai testé pour voir qu'est-ce comment quelles étaient leurs réactions personne n'a eu l'air surpris parce que je connais leur niveau très bon niveau de grammaire (motif-parce que) donc ça ils doivent le connaître par cœur + c'était pas tellement une surprise pour moi bon je l'ai quand même dit parce qu'il fallait que je le dise mais normalement je le fais de façon formelle d'abord et puis après on fait l'application + avec des exercices et puis après on sort des exercices pour l'application libre on va dire ou à l'écrit ou à l'oral + hein mais ça c'est une façon un peu particulière parce que le niveau est très élevé (motif-parce que) donc j'ai confiance je sais qu'ils vont le savoir par coeur sauf que bon alors là maintenant on doit aller euh on doit l'utiliser passer à l'étape de de l'emploi + c'est pour ça que je me permets de le faire comme ça mais ça c'est exceptionnel + pour des niveaux élevés

On remarque que C. dénomme son action (« une application pratique »), exprime ses intentions ou motif-en-vue-de («pour expliciter un point important de grammaire»), mais relève aussi ce qui l'a guidée dans le moment de l'interaction et ce qui a présidé à ses choix et à sa pratique (ou motif-parce-que) : une bonne connaissance de son public («parce que je connais leur très bon niveau de grammaire ») et un calcul interprétatif lui-même étayé par un mécanisme de réciprocité des évaluations («j'ai confiance je sais qu’ils vont le savoir par cœur $\gg)$.

Elle met ses intentions et ses motifs en contrepoids avec un déroulement schématique et prototypique de ce type d'action didactique («normalement je n'utilise pas..., normalement je fais de façon formelle ... et puis après...et puis après ») et avec un principe de la doxa pédagogique (« je l'ai quand même dit parce qu'il fallait que je le dise »), consistant à exposer la règle, quel que soit la tournure que prend l'activité didactique. C'est en quelque sorte l'expérience passée qui est invoquée et qui nourrit le faire de l'enseignante. Celle-ci rend le professeur conscient de la variabilité des pratiques de transmission de classe, ici selon le niveau de compétence des apprenants. En vertu de cette connaissance constituée par le stock d'expériences accumulées, l'enseignante déclenche des actions en-vue-de. C'est le passé de l'acteur qui entre en jeu pour la mise en jeu de l'interaction.

Les deux strates de motifs de l'action se combinent étroitement : le motif parce-que appuyé sur une connaissance basée sur ce que l'enseignante a observé et expérimenté et le motif envue-de, fortement marquée dans une action d'enseignement dont la principale raison d'être est de provoquer un accroissement du savoir de l'autre (motif en-vue-de).

La signification et l'unité de son action s'élabore, dans et par le discours, à partir de l'articulation, voire de l'appariement de ces ressources (motifs, intentions, connaissances du public, principes non dérogeables, etc.) tantôt stabilisées, tantôt indexicales. 


\section{Pour conclure : le «clair-obscur de l'interaction », son potentiel interprétatif et ses limites}

Notre contribution visait à montrer que l'analyse linguistique des processus interactionnels en situation d'enseignement des langues étrangères pouvait s'enrichir d'autres apports comme l'analyse de l'activité ou la sociologie et ouvrir sur d'autres connaissances de l'action enseignante, selon le principe du «clair-obscur» dans le sens où nous aurions deux versants complémentaires de l'analyse de l'interaction. Grâce aux outils interprétatifs de la linguistique des interactions et de l'acquisition, le chercheur peut donner une lecture claire des phénomènes interactionnels. Mais l'éclairage des verbalisations sur cette interaction nous montre que pourtant des zones sombres y subsistent. Ainsi, les intentions, motifs, connaissances, cadres d'interprétation personnels, demeurant obscurs dans l'interaction « réalisée » apparaissent nettement lorsqu'on interroge l'acteur professeur.

La compréhension des processus d'interaction in situ implique la compréhension de la complexité de cette interaction, son caractère pluriel et multidimensionnel, ses éléments d'influence plus ou moins cachés (socio-affectifs, psychologiques, socio-historiques). De plus, l'enjeu de compréhension se double d'un enjeu de formation : la connaissance et la mise en évidence de certains « ressorts» de l'interaction permet d'espérer de pouvoir les travailler, les solliciter en formation avec des enseignants.

Toutefois, s'appuyer sur les propriétés heuristiques des interactions implique certaines précautions d'ordre méthodologique, en ce qui concerne en particulier les conditions d'interprétation de l'action et de l'interaction. Tout d'abord, cette mise en interaction et en dialogue de différents points de vue ne doit pas occulter le caractère (re)construit de ces points de vue, celui de l'acteur-enseignant avisé comme celui de l'analyste. Ensuite, ce sont les différents niveaux de connaissance et d'interprétation mobilisés par les différents acteurs qu'il faut garder à l'esprit. Pour le citadin (ou l'enseignant expérimenté qu'on observe dans sa classe), les actions se déroulent sur le mode de l'évidence, mais laisse l'analyste, cherchant à éclairer le fonctionnement des interactions en classe, dans une position d'extériorité. En revanche, lorsque l'enseignant devient observateur de son activité, l'écart de statut entre le praticien et le chercheur semble se réduire. Il existerait ainsi pour la connaissance de l'agir professoral, via des interactions et des verbalisations, un continuum d'attribution de significations de l'action didactique et des situations d'enseignement entre enseignantobservé, enseignant-observateur et analyste. Ce continuum non seulement s'étale sur l'axe temporel de la successivité, mais chacune de ses étapes possède une temporalité propre. Mais il implique pour le chercheur de croiser les données, de scruter les récurrences pour s'assurer de leur fondement. C'est ainsi qu'il peut espérer donner un éclairage sur ce qui reste dans le « clair-obscur », l'action humaine.

\section{Références bibliographiques}

Ali Bouacha A. (1984). Le discours universitaire, la rhétorique et ses pouvoirs. Bern : Peter Lang.

Allwright, D. (1988). Observation in the language classroom. London, New-York: Longman.

Amigues, R., Faïta, D. \& Saujat, F. (2004). L'autoconfrontation croisée : une méthode pour analyser l'activité enseignante et susciter e développement de l'expérience professionnelle. Bulletin de psychologie (pp. 41-44). Tome 57 (1). Paris.

Bange, P. (1992). À propos de la communication et de l'apprentissage en L2, notamment dans ses formes institutionnelles. AILE 1 (pp. 53-85). Paris : ENCRAGES. 
Bigot, V. (2002). Lieux et modes de construction de la relation interpersonnelle en contexte didactique. Analyse d'interactions verbales en cours de français langue étrangère. Thèse pour le doctorat de Didactologie des Langues et des Cultures. Université Paris III-Sorbonne Nouvelle.

Bigot, V. \& Cicurel, F. (Ed.) (2005). Les interactions en classe de langue. Le français dans le monde. Recherches et Applications. Paris : CLE International.

Bouchard, R. (éd.). (1984). Les échanges langagiers en classe de langue. Grenoble : ELLUG.

Bouchard R. (2005). Le "cours », un évènement oralographique structuré : étude des interactions pédagogiques en classe de langue et au-delà... Le français dans le monde. Recherches et Applications (pp. 64-74). Paris : CLE International.

Cadet, L. (2004), Entre parcours d'apprentissage et formation à l'enseignement : le journal de bord d'apprentissage, analyse d'un objet textuel complexe. Thèse pour le doctorat de Didactique des Langues et des Cultures, université Paris III-Sorbonne Nouvelle.

Cicurel, F. \& Moirand, S. (Ed.). (1986). Discours didactiques et didactique des langues. Études de linguistique appliquée 61. Paris : Didier.

Cicurel, F. \& Doury, M. (Ed.) 2001. Interactions et discours professionnels: usages et transmission. Carnets du Cediscor 7. Paris : Presses de la Sorbonne Nouvelle.

Cicurel, F. (1988). Fiction et mise en scène dans un cours de langue, LEND 1, Année 17 (pp. 18-31). Edizioni Scolastiche Bruno Mondadori.

Cicurel F. (2007). L'agir professoral, une routine ou une action à haut risque. In Plazaola Giger, I. \& Stroumza, K. (Ed), Paroles de praticiens et description de l'activité (pp. 17-36). Bruxelles : De Boeck.

Clot, Y. (1999). La fonction psychologique du travail. Paris : PUF.

Clot, Y. (2001). Clinique du travail et action sur soi. In Baudoin, J.-M. \& Friedrich, J. (Ed.), Théories de l'action et éducation (255-304). Bruxelles : De Boeck.

Clot, Y. \& al. (2001). Entretiens en autoconfrontation croisée : une méthode en clinique de l'activité, Education permanente 146, (pp. 17-25).

Coste, D. (1984). Les discours naturels de la classe, Le français dans le monde 183 (pp. 1625). Paris : Hachette-Larousse.

De Pietro, J.-F., Matthey, M. \& Py, B. (1989). Acquisition et contrat didactique : les séquences potentiellement acquisitionnelles de la conversation exolingue. In Weil, D. \& Fugier, H. (Ed.), Actes du $3^{\text {ème }}$ colloque international de linguistique (pp. 99-124). Strasbourg : Université L. Pasteur.

Filliettaz, L. (2002). La parole en action. Éléments de pragmatique psycho-sociale. Québec: Éditions Nota Bene.

Flanders, N. A. (1960). Interaction analysis in the classroom : a manual for observers. Ann Arbor : University of Michigan Press.

Gajo, L. \& Mondada, L. (2000). Interactions et acquisitions en contexte. Fribourg : Éditions Universitaires de Fribourg.

Ginabat, H. (2006). La planification dans l'agir enseignant. Mémoire pour le Master de recherche Didactique du français et des langues, Université Paris III-Sorbonne nouvelle.

Goffman, E. (1991). Les cadres de l'expérience. Paris : Éditions de Minuit.

Guérin, F. et al. (Ed). (1997). Comprendre le travail pour le transformer. La pratique de l'ergonomie. Lyon : Éditions de l'ANACT.

Ishikawa, F. (2002). L'interaction exolingue. Analyse de phénomènes métalinguistiques, continuité et discontinuité entre situation d'enseignement/apprentissage et situation " naturelle ». Japon : Editions Shumpûsha.

Kerbrat-Orecchioni, C. (1990, 1992, 1994). Les interactions verbales, Tome I, II et III. Paris : A. Colin.

Lahire, B. (2001). L'homme pluriel. Les ressorts de l'action. Paris : Hachette littératures. 
Moirand, S. (1993). «Autour de la notion de didacticité ». Les Carnets du Cediscor 1. Paris : Presses de la Sorbonne nouvelle.

Mondada, L. (2001). Pour une linguistique interactionnelle. Marges Linguistiques 1. SaintChamas : M.L.M.S. Éditeur.

Plazaola Giger, I. (2007). La leçon racontée par l'enseignant : analyse de l'agir textualisé. In Plazaola Giger, I. \& Stroumza, K. (Ed). 2007. Paroles de praticiens et description de l'activité (pp. 37-56). Bruxelles : De Boeck.

Postic, M. (1977). Observation et formation des enseignants. Paris : PUF.

Py, B. (1993). L'apprenant et son territoire : système, norme et tâche, AILE 2 (pp. 9-24). Paris : ENCRAGES.

Rivière, V. (2006). L'activité de prescription en contexte didactique. Analyse psycho-sociale, sémio-discursive et pragmatique des interactions en classe de langue étrangère et seconde. Thèse pour le doctorat en didactique des langues et des cultures. Université Paris 3-Sorbonne Nouvelle.

Schütz, A. (1987). Le chercheur et le quotidien. Paris : Klincksieck.

Schütz, A. (1998). Éléments de sociologie phénoménologique. Paris : L'Harmattan.

Vasseur, M.-T. (2005). Rencontres de langues. Question(s) d'interaction. Paris : Didier.

Van Lier, L. (1988). The Classroom and the Language Learner, London, New-York: Longman. 